\title{
Statistically Valid Graph Representations of Scale-Space Geometry
}

\author{
Tomoya Sakai and Atsushi Imiya \\ Institute of Media and Information Technology, Chiba University, Japan \\ \{tsakai,imiya\}@faculty.chiba-u.jp
}

\begin{abstract}
This paper presents a statistical scale-selection criterion for graph representations derived from differential geometric features of a greyscale image in a Gaussian scale space. The image gradient in scale space derives hierarchical and topological relationships among the bright and dark components in the image. These relationships can be represented as a tree and a skeleton-like graph, respectively. Since the image at small scales contains invalid geometric features due to noise and numerical errors, a validation scheme is required for the detected features. The presented scale-selection criterion allows us to identify the valid features used for the graph representations with statistical confidence.
\end{abstract}

\section{Introduction}

We propose graph representations of an greyscale image and their statistical validation scheme wholly integrated in a Gaussian scale-space framework. The scale-space theory 1234 is a mathematical framework for multiscale image representation, which clarifies hierarchical relationships and topological relationships among geometric features of the image such as critical points and isophotes [5]61011], and separatrices [8]. Graph representations of these relationships among the image features are available for abstracting the structure of the image.

In general, the image analyses using a pre-smoothing filter suffer from the selection of the filter bandwidth. This problem can be interpreted as selection of the scale at which invalid features of the image due to noise are suppressed without smoothing away the valid image features for the analyses. Therefore, we require a scheme to validate the image features.

In this paper, we present a scale-selection criterion for two graph representations of an image: (i) scale-space tree for the hierarchical structure of the image and (ii) scale-space skeleton for the topological structure of the image. These graph representations are derived from the image gradient in scale space. Our scale-selection criterion is based on a statistical approach similar to scale-based clustering methods [12[13/14 15]. 


\section{Scale Space Basics}

\subsection{Gaussian Scale Space}

We define the image as a nonnegative scalar function of one or more variables whose domain is the extended real space.

Definition 1. An image is defined as a nonnegative scalar function $f(\boldsymbol{x}), \boldsymbol{x} \in$ $\overline{\mathbb{R}}^{d}$.

Here, $\overline{\mathbb{R}}^{d}$ denotes $d$-dimensional extended real space, which includes a point at infinity. Although the domain of a greyscale image is practically bounded within a limited area or volume, we embed such an image in the extended real space to apply our scale-space theory.

The scale space is known as a set of blurred images derived from conversion of a scale or an inverse resolution of the original image $f(\boldsymbol{x})$. A one-parameter family of positive function $f(\boldsymbol{x}, \tau)$ is obtained from the blurring filter with a single control parameter $\tau$. Axiomatic derivation of the Gaussian kernel for the scale-space filtering was proposed by Witkin [1] and Koenderink 2].

$$
f(\boldsymbol{x}, \tau)=G(\boldsymbol{x}, \tau) * f(\boldsymbol{x})
$$

Here, $G(\boldsymbol{x}, \tau)$ is an isotropic Gaussian function

$$
G(\boldsymbol{x}, \tau)=\frac{1}{\sqrt{4 \pi \tau}^{d}} \exp \left(-\frac{|\boldsymbol{x}|^{2}}{4 \tau}\right) .
$$

The Gaussian kernel in Eq. (2) is normalised so that the scale-space image $f(\boldsymbol{x}, \tau)$ satisfies an isotropic linear diffusion equation

$$
\frac{\partial}{\partial \tau} f(\boldsymbol{x}, \tau)=\Delta f(\boldsymbol{x}, \tau), \quad f(\boldsymbol{x}, 0)=f(\boldsymbol{x}) .
$$

In this study, we deal with the Gaussian scale space in the extended real scale and space. The point at infinity and the infinite scale play essential roles in the structural analysis of the image in scale space.

Definition 2. The Gaussian scale-space image $f(\boldsymbol{x}, \tau),(\boldsymbol{x}, \tau) \in\left(\overline{\mathbb{R}}^{d}, \overline{\mathbb{R}}^{+}\right)$is the convolution of the d-dimensional original image $f(\boldsymbol{x}) \geq 0$ with the isotropic Gaussian kernel $G(\boldsymbol{x}, \tau)$.

The Gaussian scale-space image $f(\boldsymbol{x}, \tau)$ is a diffused version of the original image $f(\boldsymbol{x})$ in the infinite domain. As the features of the original image are reduced with increasing scale, the structure of the image is simplified.

\subsection{Differential Geometric Features of Scale Space Image}

Critical Points Critical points are well-defined feature points of the scale-space image $f(\boldsymbol{x}, \tau)$ in the sense of the differential geometry. 
Definition 3. The critical points are defined as points where the spatial gradient vanishes.

$$
\{(\boldsymbol{x}, \tau) \mid \nabla f(\boldsymbol{x}, \tau)=\mathbf{0}\}
$$

A regular critical point has a nonzero determinant of the Hessian matrix $\boldsymbol{H}=$ $\nabla \nabla^{\top} f(\boldsymbol{x}, \tau)$. The regular critical points can be classified into $d+1$ types based on the combination of signs of the eigenvalues of $\boldsymbol{H}$. We denote the set of signs of the eigenvalues as $( \pm, \pm, \ldots, \pm)$. For example, if $d=2$ we have three types of critical points: local maximum $(-,-)$, saddle $(+,-)$, and local minimum $(+,+)$.

The critical points can be considered as geometric cues. The local maxima are representative of bright image components. The local minima correspond to dark cavities in the image. Note that the point at infinity is a hidden local minimum for a positive image. The local minimum at infinity represents the dark background of the positive image. The saddle points reside between the local extrema, and imply connections between them.

Critical Curves Observing these critical points in the scale space, we see their trajectories across scales called the critical curves.

Definition 4. The critical curves are the trajectories of critical points in scale space.

Figure 1(a) shows an example of the critical curves in scale space. The critical curves are also classified as local maximum curves, saddle curves, and local minimum curves. Since a saddle point exists between local extrema, disappearance of a local extremum with increasing scale is always accompanied by that of the saddle point. The local extrema are annihilated when they meet the saddle points with increasing scale. The annihilation point is a singular point where at least one of the eigenvalues of $\boldsymbol{H}$ is zero. Therefore, a local extremum curve and a saddle curve can share the singular point in the scale space. The connections of the critical curves in scale space indicate topological relationships among the local extrema and saddle points.

Figure Field. In thermal physics, the diffusion equation describes transmission of heat. The solution and its spatial gradient are the transition of temperature distribution and the flow of heat. In the scale space of an image, $f(\boldsymbol{x}, \tau)$ and its spatial gradient are analogically the transition of the distribution of image intensity and the flow of image intensity. Therefore, the spatial gradient field of the scale-space image $f(\boldsymbol{x}, \tau)$ and its field curves are called the figure field and the figure-flow curves, respectively [5].

Definition 5. The figure field $\boldsymbol{F}$ in the isotropic space at a scale $\tau$ is defined as the negative of the gradient vector field of the scale-space image.

$$
\boldsymbol{F}=-\nabla f(\boldsymbol{x}, \tau)
$$

Definition 6. The figure-flow curves are the directional lines of the figure field.

Figure 1(b) illustrates an example of the figure-flow curves of a two-dimensional scale-space image at a fixed scale. The local maxima, local minima, and saddle points are the sources, drains, and confluent points of the flow of image intensity. 


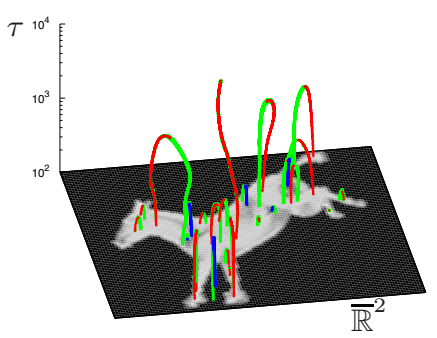

(a)

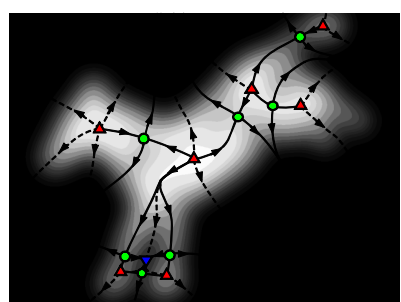

(b)

Fig. 1. Critical curves and figure-flow curves. (a) Critical curves in scale space for an image of a kicking horse [16. (b) Figure-flow curves of the image at $\tau=400$. The solid curve indicates the separatrix. The up triangle, down triangle, and circle indicate the local maximum, local minimum, and saddle point, respectively.

Separatrices. The topological relationships among the critical points can be analysed by the figure-flow curves. In two-dimensional case, a saddle $(+,-)$ has a pair of each inward and outward flow curves called the separatrices. A separatrix of the inward flow curves connects between local maxima of $f(\boldsymbol{x}, \tau)$, i.e., the sources of the flow.

\section{Graph Representations of Scale-Space Geometry}

\subsection{Scale-Space Tree}

The critical curves in scale space provides a topological relationships between the critical points. It is suggested that the singular point shared by a pair of critical curves is connected to another local extremum by the so-called antidirectional figure-flow curve at the scale of annihilation as shown in Fig. 2(a) 11. Since the figure-flow curve is the path of the flow of image intensity, the antidirectional figure-flow curve indicates the source or drain of the flow to or from the singular point. Consequently, hierarchical relationships among the critical points are exhibited in the scale space by the critical curves and the antidirectional figure-flow curves.

The hierarchical relationships are symbolically expressed as a tree. We present an algorithm for bottom-up construction of a tree $T$.

\section{Algorithm I: Scale-Space Tree}

1. Set nodes for critical points at an initial scale $\tau=\tau_{0}$ to be the leaves of $T$.

2. Diffuse the image $f\left(\boldsymbol{x}, \tau_{n-1}\right)$, yielding $f\left(\boldsymbol{x}, \tau_{n}\right)$.

3. Detect annihilation events of local extrema and saddles within $\tau_{n-1}<\tau \leq \tau_{n}$ by examining links of the critical points between the subsequent scales $\tau_{n-1}$ and $\tau_{n}$.

4. For each detected annihilation event:

i Add to $T$ a new node with two branches leading to nodes for the local extremum and saddle involved in annihilation. 


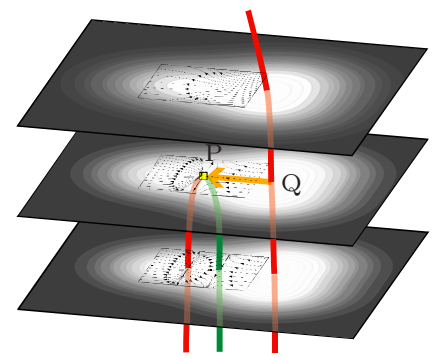

(a)

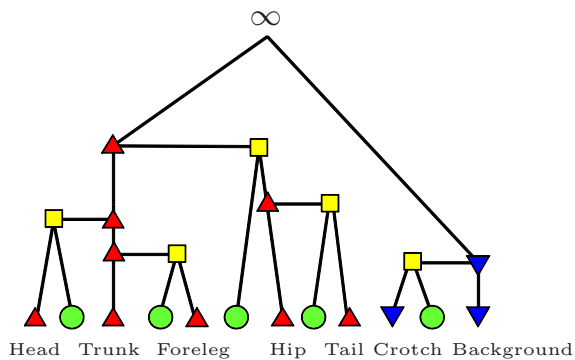

(b)

Fig. 2. Graph representation of hierarchical relationships among the critical points. (a) The singular point $P$ is connected to the local maximum $Q$ by the antidirectional figure-flow curve, which indicates subordinate relationship between them. (b) A few levels of scale-space tree of the kicking horse image in Fig. 1] The square indicates the singular point. $\infty$ at the root indicates the virtual annihilation.

ii For the annihilation event of the local maximum, maximise $f\left(\boldsymbol{x}, \tau_{n}\right)$ using the annihilation point for the initial point.

iii For the annihilation event of the local minimum, minimise $f\left(\boldsymbol{x}, \tau_{n}\right)$ using the annihilation point for the initial point. Regard the annihilation point as having a connection to a local minimum at infinity if minimisation results in a point on the image boundary.

iv Add to $T$ a new node with two branches. One branch is attached to the node for annihilation point. The other branch is attached to a node for the local extremum determined by the maximisation or minimisation.

5. Return to step 2 until there remains no saddle point.

6. Add to $T$ a node for the virtual annihilation point of one remaining local maximum and the local minimum at infinity.

An example of the scale-space tree is shown in Fig. 2(b).

We store in the nodes of $T$ the scales of annihilation. The scales of annihilation can be used for a statistical test of the validity of the critical points, which is discussed in Section 4. Note that Algorithm I is not designed to detect the creation event 7910] because we are interested in the hierarchical relationships among the critical point at the initial scale $\tau_{0}$.

\subsection{Scale-Space Skeleton}

We present a novel graph representation of topological relationships among local maxima of the image. Since the local maxima are the sources of the flow of image intensity with respect to scale, the critical curves of local maxima and the antidirectional figure-flow curves indicate how the distributed image intensity is inherited from coarse to fine. At the coarsest scale, one remaining local maximum represents the total image intensity. As the scale decreases, the local maxima appear when the image intensity is supplied by the existing local 


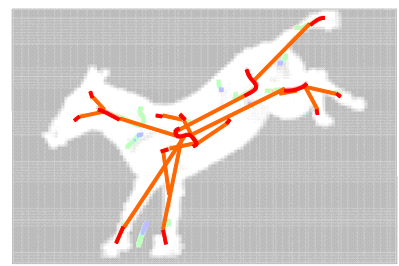

Fig. 3. Scale-space skeleton of the kicking horse

maxima through the antidirectional figure-flow curves. The inheritance of the image intensity is symbolically expressed by a tree with nodes related to the local maxima embedded in the scale-space tree.

Geometrically, the critical curves $\left\{(\boldsymbol{x}, \tau) \mid \nabla f(\boldsymbol{x}, \tau)=\mathbf{0}, \boldsymbol{x} \in \overline{\mathbb{R}}^{d}, \tau \geq \tau_{0}\right\}$ in scale space can be projected onto the space $\overline{\mathbb{R}}^{d}$ as one-dimensional manifolds $\left\{\boldsymbol{x}(\tau) \mid \boldsymbol{x}(\tau) \in \overline{\mathbb{R}}^{d}, \tau \geq \tau_{0}\right\}$. Here, $\tau_{0}$ is an arbitrary scale. The antidirectional figure-flow curves at $\tau \geq \tau_{0}$ can also be projected onto $\overline{\mathbb{R}}^{d}$ as the curves, which supply the linkage between the manifolds $\{\boldsymbol{x}(\tau)\}$. We call the resulting graph the scale-space skeleton, which exhibits trajectories of the representative points of the flow of image intensity. The nodes of the graph represent critical points at the scale $\tau_{0}$, and all singular points and their source points. The edges of the graph are the projected critical curves and antidirectional figure-flow curves. Figure 3 is an illustration of the scale-space skeleton. A skeleton-like representation of the image is obtained from the scale space.

\section{Statistical Selection of Scale}

In general, most of the image analyses using Gaussian filtering suffer from the selection of the filter bandwidth, i.e., the scale. The image loses its geometric features at large scales. The detected features, however, are invalid at small scales because the geometric features are infected by unfiltered noise. The differential operations enhance the noise, especially. As the result, the detected features are so random and experimentally less reproducible. We require a validation scheme to identify the image features with the statistically significant reproducibility.

Clustering methods of data points using scale space [121314 give us a hint of the validation of the geometric features of image. In these clustering methods, the data clusters are related to the modes of the probability density function (PDF) of the data points. The PDF is estimated by taking the sum of the Gaussian kernel functions at the data points, which satisfies the scale-space axioms. The cluster validity is attributed to that of the estimation of modes, or the local maxima of the estimated PDF. We have proposed a statistical criterion to identify the valid clusters by the life of mode [15]. The life is defined as 


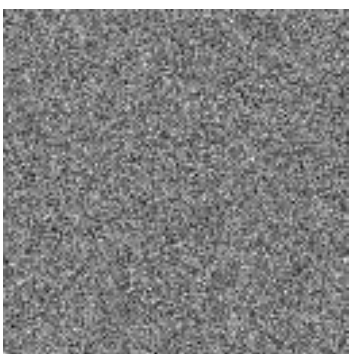

(a)

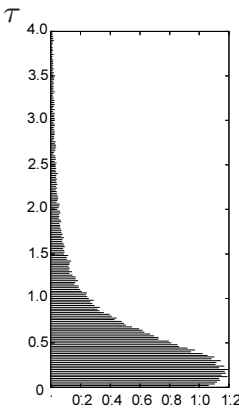

Relative frequency

(b)

Fig. 4. Noise image and life histogram for local maxima. (a) The noise image has uncorrelated random pixel values. (b) The life histogram shows relative frequency of scale at which the local maxima of the noise image are annihilated as Gaussian blurring proceeds.

the terminating scale of the critical curve of the mode, which is equivalent to the scale of the singular point. We showed that the uniformly distributed data points present a Weibull-like unimodal distribution of the life. The valid cluster can be defined as a cluster with a statistically significant life out of this unimodal distribution. Consequently, the cluster validation is achieved by the statistical rejection method using the unimodal life distribution. The estimated PDF for the uniformly distributed data points is interpreted as an image of the background noise in image analyses.

For the purpose of the statistical selection of the scale for the graph representations in Section 3 , we investigate experimentally the distribution of the life of the critical points detected in a two-dimensional noise image with uncorrelated random pixel values as shown in Fig. 4(a). We averaged the frequencies of lives over a hundred of noise images with $128 \times 128$ pixels. Figure 4(b) is the averaged histogram of life for local maxima. The obtained life histogram shows an unimodal shape. The critical points with significantly large values of life out of this unimodal distribution can be identified to be valid, because they are distinguishable from the invalid critical points of the noise image.

We can set a critical value of the scale to judge the critical points valid or invalid. Although the computation of such a critical scale requires the parametric model of the life distribution in the strict sense of statistics, the critical scale can be roughly evaluated by the peak and decaying form of life histogram. The peak is found at a small scale relative to the outlying lives if the image contains valid critical points. According to our experimental result, a critical point with a life which is more than a hundred times greater than the peak can be considered to be valid with the statistical confidence level $\alpha>98.9 \%$ under the assumption of uncorrelated random pixel values of a two-dimensional image. 


\section{Concluding Remarks}

The spatial gradient of the Gaussian scale-space image clarifies the hierarchical and topological structure of the image. We presented a scale-selection criterion for the scale-space tree and the scale-space skeleton. These graph representations consist of the critical points of which validity is statistically guaranteed above the selected scale. Our image analysis is wholly integrated in the Gaussian scale-space framework. The scale-space theory has considerable potential for combining multiscale feature detection and statistical validation for image representations.

\section{References}

1. Witkin, A.P.: Scale space filtering. In: Proc. of 8th IJCAI, pp. 1019-1022 (1986)

2. Koenderink, J.J.: The structure of images. Biological Cybernetics 50, 363-370 (1984)

3. Lindeberg, T.: Scale-Space Theory in Computer Vision. Kluwer, Boston (1994)

4. Weickert, J., Ishikawa, S., Imiya, A.: Linear Scale-Space has First been Proposed in Japan. Journal of Mathematical Imaging and Vision 10, 237-252 (1999)

5. Zhao, N.-Y., Iijima, T.: A theory of feature extraction by the tree of stable viewpoints. IEICE Japan, Trans. D. J68-D, 1125-1135 (1985) (in Japanese)

6. Lifshitz, L.M., Pizer, S.M.: A multiresolution hierarchical approach to image segmentation based on intensity extrema. IEEE Trans. Pattern Analysis and Machine Intelligence 12(6), 529-540 (1990)

7. Griffin, L.D., Colchester, A.: Superficial and deep structure in linear diffusion scale space: Isophotes, critical points and separatrices. Image and Vision Computing 13(7), 543-557 (1995)

8. Olsen, O.F., Nielsen, M.: Generic events for the gradient squared with application to multi-scale segmentation. In: ter Haar Romeny, B.M., Florack, L.M.J., Viergever, M.A. (eds.) Scale-Space 1997. LNCS, vol. 1252, pp. 101-112. Springer, Heidelberg (1997)

9. Florack, L.M.J., Kuijper, A.: The topological structure of scale-space images. Journal of Mathematical Imaging and Vision 12(1), 65-79 (2000)

10. Kuijper, A., Florack, L.M.J., Viergever, M.A.: Scale space hierarchy. Journal of Mathematical Imaging and Vision 18(2), 169-189 (2003)

11. Sakai, T., Imiya, A.: Gradient structure of image in scale space. Journal of Mathematical Imaging and Vision 28(3), 243-257 (2007)

12. Roberts, S.J.: Parametric and non-parametric unsupervised cluster analysis. Pattern Recognition 30(2), 261-272 (1997)

13. Nakamura, E., Kehtarnavaz, N.: Determining number of clusters and prototype locations via multi-scale clustering. Pattern Recognition Letters 19(14), 1265-1283 (1998)

14. Leung, Y., Zhang, J.-S., Xu, Z.-B.: Clustering by scale-space filtering. IEEE Trans. on Pattern Analysis and Machine Intelligence 22(12), 1396-1410 (2000)

15. Sakai, T., Imiya, A., Komazaki, T., Hama, S.: Critical scale for unsupervised cluster discovery. In: Perner, P. (ed.) MLDM 2007. LNCS (LNAI), vol. 4571, pp. 218-232. Springer, Heidelberg (2007)

16. Muybridge, E.: Animal Locomotion (1887) 